\title{
475176 - THE VISCOSITY OF HYDROXY-ETHYL STARCH
}

Clifton Johnston, $\mathrm{PhD}^{2}$, Jacqueline Flewitt, BEng, MSc. ${ }^{2}$, Kogan Lee, BEng (in progress) $^{2}$, Andrew Walker, BSc, MBIS ${ }^{1}$, Gary Dobson, M.D.,C.M. ${ }^{1}$

1. Anesthesia, University of Calgary, Calgary, AB, Canada

2. Mechanical Engineering, University of Calgary, Calgary, AB, Canada

Introduction: There are two hydroxyl-ethyl starches (HES) available for volume expansion in Canada: HES 260/0.45 and HES 130/0.4. While information regarding their pharmacokinetic(1) and risk/benefit profiles is available(2), there is no published data on their respective viscosities.

Methods: The viscosity for each HES was determined at concentrations of 1.0, 0. 75, 0.5, 0.25 and 0.125 the original. For each concentration the viscosity was evaluated at temperatures of 21, 25, 30 and 37 degrees Celsius. Three measurements were made for each concentration and temperature. Serial dilutions were with normal saline. The use of an incubator assured a constant temperature throughout the course of a measurement. Saline and tap water were used as controls. The viscosity was measured using a capillary viscometer, with all measurements taking greater than 100 seconds.

Results: There is a non-linear relationship between the HES concentration and viscosity (Fig. 1). This effect was greatest for the $10 \%$ HES and was attenuated by increased temperature. At concentrations of less than $5 \%$, the HES viscosity is $2 / 3$ to $1 / 3$ that of whole blood. The kinematic viscosity (viscosity / density) demonstrated a similar relationship.

Discussion: The non-linear relationship between concentration and viscosity suggests the development of large molecular complexes at the lower temperatures. In spite of the published differences in their mean molecular weights, at body temperature and clinically relevant concentrations (1-5\%), the differences between the two HES solutions are minimal. Whether these small differences translate into clinically important differences in plasma viscosity and shear stress is unknown.

References: (1) Jungheinrich C, Neff TA. Pharmacokinetics of hydroxyethyl starch. [Review] [64 refs]. Clinical Pharmacokinetics 44(7):681-99, 2005. (2) Boldt J. Do plasma substitutes have additional properties beyond correcting volume deficits? [see comment]. [Review] [96 refs]. Shock 25(2):103-16, 2006 Feb. 


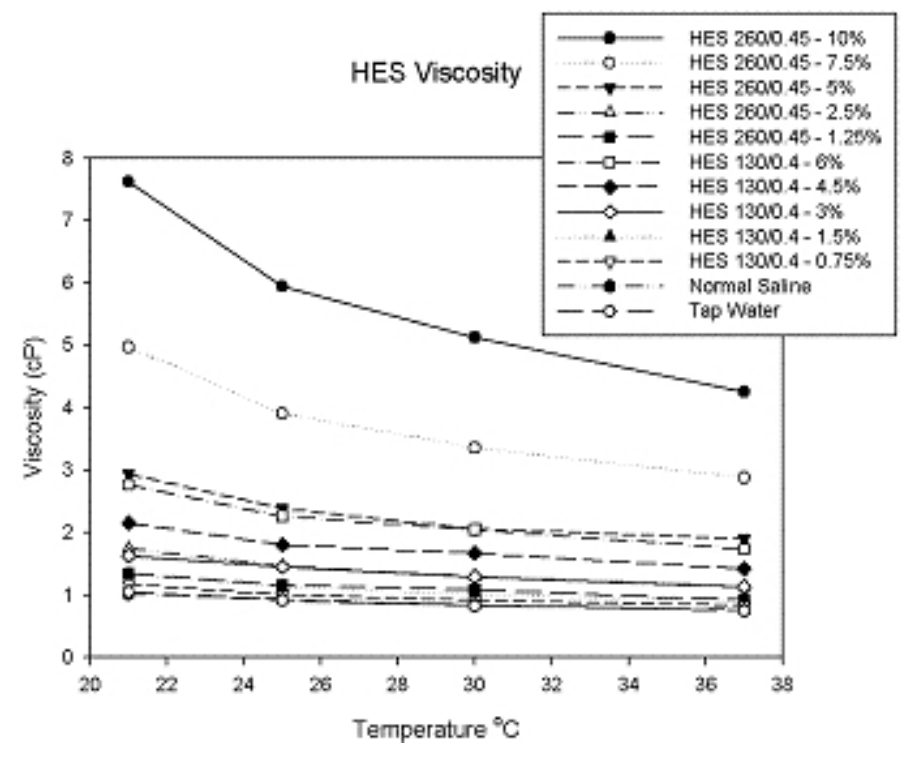

